\title{
Persönliche Erfahrungen mit Inhaftierten, die als "gefährlich« galten.
}

Martin Hagenmaier

$\mathrm{D}$ ie Durchführungsverordnung zur nachträglichen Sicherungsverwahrung in Schleswig - Holstein, einem Land, das sich gegen diese Regelung ausgesprochen hatte, spricht Bände: Anträge werden durch eine schlichte Prüfung der Häufigkeit von früheren Verurteilungen ausgelöst und die Prüfung betrifft künftig alle Inhaftierten, nicht nur besonders herausragende »Schwerkriminelle ${ }^{1}$. Die nachträgliche Verwahrung schwebt somit wie ein Damoklesschwert über allen mehrfach verurteilten mit langen Freiheitsstrafen. Es wird sich zeigen, ob kluge Vollstreckungsgerichte dem Grenzen setzen. ${ }^{2}$ Was aber besagen solche Erkenntnisse für den Umgang mit Männern, deren Leben in der Gesellschaft und in ihren kleineren Subsystemen für einzelne ihrer Mitglieder eine (körperliche und / oder seelische) Gefahr bedeuten? Um der Antwort näher zu kommen, berichte ich über seelsorgerlichen Umgang mit Männern, denen das Etikett gemeingefährlich nach allen menschlichen Übereinkünften, aber auch vor Gericht zustand / zusteht.

a) Klaus Grabowski wurde dadurch sehr bekannt, daß er in einem Lübecker Gerichtssaal von der Mutter des Kindes, das er getötet hatte, mit sechs Schuß in den Rücken erschossen wurde. Dieser Mann hatte im Lübecker Gefängnis eine Art Bekehrung zum christlichen Glauben erlebt. Das Urteil wegen einer Sexualstraftat erging dann nach dem $§ 21,63$ StGB als Einweisung in die forensische Psychiatrie. Hier schätzte sich der Patient Grabowski selbst als gemeingefährlich ein und hoffte auf den Glauben und auf die bevorstehende Kastration als »Heilung «. Seine Art von Gläubigkeit wirkte sehr »hochgefahren « und wenig realitätsgeeignet. Dennoch band sie Energien durch eifrigen Einsatz in der Missionierung anderer Patienten. Zudem ergab sich durch die Bekehrung eine soziale Einbindung in eine strenggläubige Gemeinde. Klaus Grabowski war sogar fähig, sich mit seinem bis dahin geführten - nach seiner Aussage verpfuschten - Leben auseinanderzusetzen. Er wies ausdrücklich seinen sozialen Umwelten keine Schuld, sondern nahm sie verstehend in Schutz. Seine Eltern und seine ehemalige Frau mit den Kindern - allen habe er schlimme Schmerzen zugefügt. Möglicherweise sprach er zu wenig über das eigentliche Problem seines sexuellen Kindesmißbrauchs. Nach der Kastration wurde er relativ schnell in die Freiheit und zur ambulanten Weiterbehandlung entlassen. ${ }^{3}$ Warum er die kleine Anna tötete, konnte nicht ermittelt werden. Man könnte die These aufstellen, die abweichende Sexualität habe er mit Abspaltung dieser »verbotenen Anteile « bearbeitet, was ihre plötzliche Wiederkehr erklären könnte.

b) Dieter F. erlebte die forensische Psychiatrie als Sexualstraftäter ( $§ 176$ I StGB) und etwas zu mustergültiger Patient. In Gesprächen wirkte er freundlich, aber auch unfaßbar und merkwürdig unbeteiligt. Arbeiten auf der Abteilung erledigte er unglaublich akkurat. Er verbarg in Gesprächen seine Straftaten nicht, setzte sich aber damit auch nicht auseinander. Immer wieder betonte er ungefragt und nachdrücklich, er sei zu zwei Jahren Haft verurteilt und müsse danach auch aus der Psychiatrie entlassen werden. Den verurteilenden Richter rief er stets zum Zeugen an. Alle Erläuterungen, in der Psychiatrie dauere die Unterbringung bis auf weiteres, zumindest bis zum nächsten Gutachten, glitten an ihm ab. Er weigerte sich nachdrücklich, diese Situation wahrzunehmen. Ohne Ausehen auf der Abteilung kam nach einiger Zeit die Verlegung in den halboffenen Bereich in Betracht. Die erste Lockerung sollte der Gang zur Gärtnerei im Krankenhausgelände sein. Als Dieter F. dann verschwunden war, stellte sich heraus, daß er genau zwei Jahre in der Psychiatrie verbracht hatte. Er hatte sich selbst entlassen. Psychiatrisch war er offenbar unauffällig gewesen. Dieter F. brachte nach dieser Flucht auf grausame Weise ein 13jähriges Mädchen um und wurde erst Monate später durch Zufall auf dem Hamburger Dom verhaftet.

c) XY. wagte sich nicht ohne Begleitung aus seiner Gefängniszelle. Zu Gesprächen, Gruppenveranstaltungen und zum Gottesdienst wurde er gebracht. Achtzehn Jahre hatte er bereits wegen Kindesmißbrauchs hinter und zwei Jahre wegen desselben Delikts (\$176 I StGB) noch vor sich. In der Haft wurde er sechzig. Einer seiner Standardsätze hieß: »Wenn die alles gefunden hätten, müßte ich noch im Sarg hier stehen.« Er hatte zwei in (an der?) Haft gescheiterte Ehen hinter sich. Durch therapeutische Betreuung und viele Gespräche differenzierte sich sein Selbstbild. Er fand einen Zugang zu der Frage, wie sich seine sexuellen Handlungen mit Kindern anbahnen. Seine therapeutische Betreuung hält nach der Entlassung an. Er suchte auch weiter Kontakt mit anderen Betreuungspersonen aus der Haftzeit. Ein Rückfall erfolgte in vier Jahren Freiheit trotz schwerer persönlicher Probleme nicht.

d) AZ erklärte in jedem Gespräch während seiner zweiten Haft, daß er natürlich nach der Entlassung dort weitermachen werde, wo er vor der Inhaftierung aufgehört habe (§ 176 III Nr. 3. StGB). Und wenn er dafür auf die Philippinen fliegen müßte. Therapie sei nicht nötig. Gespräche waren fast unnötig. Sie endeten immer mit der Ankündigung, daß er weitermachen wird wie bisher. Das änderte sich auch nicht, als seine Mutter im Sterben lag und er infolge seines Verhaltens nicht einmal ausgeführt werden konnte. Das Verhalten als Trotzreaktion zu interpretieren, untersagte der Gefangene regelmäßig. In der folgenden Haftzeit war das Bild etwas differenzierter. AZ bedauerte sein Verhalten ein ganz klein wenig und äußerte sich weniger provokativ. Die Freiheit hält inzwischen ein Jahr an.

e) BD kam mit dem Anspruch, ein furchterregender Gewalttäter zu sein, in die Strafhaft. Er erzählte mal mit mal ohne Drogen im Kopf, wen er alles wie "weggehauen « hat (§ 223, 224 I Nr. 4. $\mathrm{StGB})$. Er gestand einmal einen Mord, wo ihm die Polizei nachwies, daß er es gar nicht gewesen sein kann. Die Gespräche mit ihm glichen eher Ringkämpfen. Ereignisse in seiner (Pflegeherkunfts-)Familie und bei seiner geschiedenen Frau versetzten ihn in massive Unruhe mit der Folge aggressiver Attacken gegen Mitgefangene und Bedienstete. Ging es ihm besser, versuchte er, Mitgefangene zu dominieren, führte Attacken im Auftrag anderer Gefangener durch und tyrannisierte Schwächere. Verständnis für ihn und andere legte er gerne als Schwäche aus. Wer in Haft ein derartiges Bild zeigt, kann nicht damit rechnen, positiv beurteilt zu werden. Dennoch durfte BD in begleiteten Ausgang gehen und wurde kurz danach sogar beurlaubt. Er wohnte im Urlaub bei seiner Freundin und dem gemeinsamen Sohn. Beim zweiten Urlaub blieb er zum Entsetzen der Freundin lange von zu Hause weg. Da er 
auch nicht rechtzeitig zum Urlaubsende zurück war, meldete sie sich bei der Polizei. Nachdem BD schon einmal wegen Waffengebrauch verurteilt war und als gefährlich galt, wurde er bei der Rückkehr durch ein SEK festgenommen. Das hat ihn »schwer beleidigt «. In einer solchen Situation wollte er nicht von seiner Freundin und seinem Sohn gesehen werden. Zwei Jahre nach der Entlassung gab es unter den Gefangenen in der JVA Gerüchte, BD habe eine Frau umgebracht. In der Tat kam er nach einiger Zeit zur Verhandlung. Der Prozeß endete mit einer Verurteilung wegen vorsätzlichen Vollrausches (§ 323a StGB), da sich der genaue Hergang des brutalen Verbrechens an der getöteten Frau nicht rekonstruieren ließ.

Die Fälle sind nicht repräsentativ, sondern beispielhaft ausgewählt. Auf alle wird der Begriff »gemeingefährlich « deshalb angewandt, weil man entweder nicht oder zu genau weiß, was passieren könnte. Als Hauptfragestellung erweist sich, wie die Gefährlichkeit auf prognostisch sichere Beine gestellt werden soll. Die Gefährlichkeit durch nachbarschaftliche Beobachtung oder Medienberichterstattung festzustellen, wird wahrscheinlich allgemein als unangemessen angesehen. Fall d) stellt seine Gefährlichkeit durch eigene Reden heraus. Fall e) fällt durch die Art seines Urteiles bereits in der Vorprüfung durch, obwohl die Karriere der Gefährlichkeit durchaus nicht nur in seinen Worten nachvollziehbar ist. Fall c) erfüllt rechtlich sämtliche Anforderungen der Prüfliste, lebt aber seit vier Jahren ohne Rückfall. Im Fall b) hätte die schlimmste der Taten nach der Flucht eventuell durch sehr viel Erfahrung in der Bedeutung von Kommunikation verhindert werden können. Das aber hängt von vielen Zufällen ab. Die betreuende Ärztin wurde der falschen Prognose wegen, die zur Lockerung führte, zu einer Geldstrafe verurteilt. $\mathrm{Ob}$ sie wirklich dafür verantwortlich $\mathrm{zu}$ machen war, erscheint unklar. Bei restriktiver Auslegung wäre nach $\S 66$ b StGB in allen Fällen nachträgliche Sicherungsverwahrung zu verhängen, weil die Verantwortung für eine Prognose zur Entlassung aus der Haft zumindest fragwürdig erscheinen müßte. Damit aber steigt die Anforderung an die Maßnahmen und Behandlungsversuche, die während der Haft angesetzt werden. Haben der Strafvollzug und die forensische Psychiatrie in Deutschland für Wiederholungstäter Karrieren dieser Art Kapazität, wissenschaftliche und therapeutische Ansätze? Nach unserer Gesetzeslage und dem dazugehörigen Menschenbild kann es eigentlich nicht vorkommen, daß Männer mit derartigen Problemen und Gefährdungen allenfalls »antherapiert « den Vollzug wieder verlassen. Die Alternative heißt jedoch nicht Sicherungsverwahrung. Sie heißt mehr Diagnose- und mehr Behandlungsmöglichkeiten im Laufe des Vollzugsgeschehens. Wenn die Alternative zur Entlassung aufgrund einer Gefährlichkeit in der Sicherungsverwahrung liegen sollte, wenn dazu die gängigen Methoden der Messung von Gefährlichkeit als Persönlichkeitsstörung und Psychopa- thie zugrundegelegt werden, wird es in absehbarer Zeit neue Formen von »lebenslänglich« geben.

Die Sichtweisen haben sich also vor allem in der grundsätzlichen Einschätzung verändert. So kann man heute im Wissenschaftsbetrieb wieder ungeniert behaupten, es gäbe Menschen, die gemeingefährlich »sind « im Sinne des Mordparagraphen: »Gemeingefährlich ist, wer ...«. Selbst kleinere Vollzugsentscheidungen werden tendenziell mit Rasterfragebogen unterlegt, um keine Einschätzungsfehler mehr $\mathrm{zu}$ machen. "Da Menschen mit antisozialer Persönlichkeitsstörung gesellschaftliche Normen und Gesetze häufig nicht akzeptieren, neigen sie zu Kriminalität und Gewalt. Im Arbeitsleben sowie in zwischenmenschlichen Beziehungen erweisen sich Betroffene in der Regel als unzuverlässig und verantwortungslos. Die Rechte anderer werden häufig mißachtet und verletzt: Menschen mit dieser Störung sind wenig selbstkritisch. Meist mangelt es ihnen an Einsicht und Schuldbewußtsein bezüglich begangenen Unrechts. «4 Wer möchte einem so klassifizierten Menschen nach bereits mehrfach erfolgter Inhaftierung eine positive Prognose ausstellen? Das gilt besonders deshalb, weil therapeutische Zugänge zu dieser Diagnosegruppe äußerst zurückhaltend beurteilt werden.

\section{Der neue Ton liegt}

1) in der Behauptung, gemeingefährliche Täter seien von denen, die diese Eigenschaft nicht haben, durch Gutachten und Profilfragebogen zu unterscheiden. Dabei spielt das wiedererstandene Persönlichkeitsmodell der Psychopathie5 eine Rolle;

2) in der Vorstellung, Kriminalität sei durch längeres Einsperren und Verwahren minimierbar, obwohl Länder mit hohen Haftquoten eher höhere Kriminalitätsraten aufweisen als die mit niedrigeren Haftquoten;

3) in der Vorstellung, Sicherheit ließe sich durch Prognosen herstellen, obwohl Menschen sich oft nicht nach Prognosen verhalten und die Fähigkeit zur treffsicheren Prognose sehr eingeschränkt vorhanden ist. Die meisten Fälle, in denen sich später »Gemeingefährlichkeit« herausstellt, waren vorher mehrfach begutachtet und prognostiziert. Bisher gibt es wenige Untersuchungen, welche die Treffsicherheit von Prognosen im Langzeitversuch nachvollziehen.

4) Wer Sicherheit durch Prognose anstrebt, unterliegt grundsätzlich der Tendenz, "Gemeingefährlichkeit« möglichst früh zu diagnostizieren. Ein solches Ausleseverfahren droht bereits in der sozialen Kategorisierung zu beginnen und vorgeburtliche Faktoren der sozialen Umwelt einzuschließen.

5) Gleichzeitig wird damit eine Logik begründet, die tendenziell Gefährlichkeit auf impulsives und risikoreiches Verhalten ausweitet.

6) Dass gemeingefährliches Verhalten von politisch oder religiös motivierten gruppenbezogenen Straftätern mit diesem Instrumentarium nicht gemessen werden kann, scheint in dieser Denk- und
Diagnoseweise niemand zu stören. Im Allgemeinen entsprechen sie keiner Psychopathielehre.

Die neue Betrachtungsweise entspricht der gesellschaftlichen Vorstellung, Leben könne und müsse durch diagnostische Auslese gesteuert werden und nicht durch Fördern, Einüben in Verbindung mit flexiblen Kontrollformen. Der Paradigmenwechsel in hochmodernen westlichen Gesellschaft ist vollzogen und beginnt sich in der Gesetzgebung auszuwirken. Wer Justiz betreibt, wird sich damit auseinandersetzen müssen, daß tendenziell immer mehr Männer nach den für sie üblichen Wiederholungstaten für sehr lange Zeit in Haft bleiben werden. Unangemessen ist daran nicht der Versuch, Beurteilungskriterien für den Umgang mit diesen schwierigen Mitmenschen zu finden, sondern die Idee, das als alleinigen Weg zur Lösung des Problems zu propagieren. Die Realität verlangt immer erfahrungsgebundene Entscheidungen, keine weltanschaulichen Lösungen. Die Sichtweise ist neu, das Problem nicht.

\section{Der Autor ist Gefängnisseelsorger in Kiel}

\section{Fußnoten:}

1 Erlaß zur Umsetzung des Gesetzes zur Einführung der nachträglichen Sicherungsverwahrung, Ministerium für Justiz, Frauen, Jugend und Familie des Landes Schleswig - Holstein vom 5.10.2004.

2 Frommel, NK 3/2004, 88 etwa stellt hohe Anforderungen an die Voraussetzung, es müsse sich um neue Tatsachen handeln. Dies würde die erkennenden Gerichte zwingen, immer dann zumindest von $\S 66$ a StGB - der vorbehaltenen Sicherungsverwahrung - Gebrauch zu machen, wenn sie Tatsachen zu beurteilen haben, aus denen sich die Gefährlichkeit des für voll schuldfähig eingestuften Verurteilten ergibt. Verneinen sie dies, präkludiert diese Entscheidung die spätere Anwendung des $\S 66 \mathrm{~b}$ StGB. $\S 66 \mathrm{~b} \mathrm{StGB}$ wäre dann nur für Altfälle oder für solche Fälle reserviert, in denen der die Gefährlichkeit begründende Hintergrund »neu« ist. Es wird sich zeigen, wie die nächste Stufe in der Umsetzung der neuen Sicherheitstechniken aussehen wird.

3 Ausführliche Falldarstellung unter seelsorgerlichem Aspekt: Hagenmaier, Martin, Seelsorge mit Sexualstraftätern, Wege zum Menschen 36/1984, S. 80ff.

4 Zitiert nach dem Manuskript der Klinik und Poliklinik für Psychiatrie und Psychotherapie der Charité in Berlin.

5 Hier spricht man von dissozialer oder antisozialer Persönlichkeitsstörung. Aufschlußreich ist jedoch die Zusammenfassung dieser Störungen unter dem Stichwort der Psychopathie, so etwa: Hart SD, CoxDN \& Hare RD (1966) The Hare PCL.SV. Psychopathy Checklist: Screening Version. MultiHealth Systems, North Tonawanda-Toronto. Die Häufigkeit von Persönlichkeitsstörungen in nordamerikanischen Gefängnissen liegt nach Hare bei $20 \%$, in der forensischen Psychiatrie bei 10 $\%$, in der Allgemeinpopulation rechnet man mit 3-7 \% bei Männern und 1-2 \% bei Frauen. Antisoziale Persönlichkeit werden angenommen zwischen 0,2 und $3 \%$, Bronisch, T., Persönlichkeitsstörungen. In: Möller, H.-J. u.a., Hg., Psychiatrie und Psychotherapie, Springer - Verlag, Berlin Heidelberg, New York 2000. Im Diagnoseschlüssel ICD 10 (F6) kommen die Persönlichkeitsstörungen unter $\mathrm{F} 60.1$ bis $\mathrm{F} 60.8$ vor 